\title{
Primer registro del género Queletia (Basidiomycota: Agaricaceae) en México
}

\author{
First record of the genus Queletia (Basidiomycota: Agaricaceae) in México
}

\author{
Olivia Rodríguez Alcántar, María de Jesús Herrera-Fonseca
}

Laboratorio de Micología, Departamento de Botánica y Zoología, Centro Universitario de Ciencias Biológicas y Agropecuarias (CUCBA), Universidad de Guadalajara, Apartado postal 1-139, C.P. 45101, Zapopan, Jalisco México.

\section{RESUMEN}

Antecedentes: Queletia es un género considerado como raro y poco estudiado. Q. mirabilis es la especie más reconocida, registrada para Europa y Norteamérica. En México diversas aportaciones se han realizado sobre Tulomastaceae, con el registro de todos los géneros excepto Queletia, el cual se cita por vez primera para el país.

Objetivo: Registrar el género Queletia para la micobiota mexicana bajo Q. mirabilis.

Métodos: Se revisó el material procedente de Nayarit, depositado en la colección micológica del herbario IBUG. Para el estudio macro- y micromorfológico se siguieron las técnicas convencionales en micología, y el uso de literatura especializada para su determinación.

Resultados y conclusiones: Se presenta la descripción morfológica de $Q$. mirabilis, fotografías del basidioma y estructuras microscópicas de importancia taxonómica. En diversos estados como Nayarit es necesario incrementar las exploraciones para un mejor conocimiento de la micobiota del país.

Palabras clave: micobiota, nuevo registro, Nayarit

\section{ABSTRACT}

Background: Queletia is a genus considered rare and little studied. Q. mirabilis is the most recognized species, registered from Europe and North America. In Mexico, several contributions have been made on Tulomastaceae, with records of all genera except Queletia, which is cited for the first time for the country.

Objective: Register the genus Queletia for the Mexican mycobiota under Q. mirabilis.

Methods: Material collected from the state of Nayarit and deposited in the mycological collection of the IBUG herbarium was studied. For the macro- and micromorphological study the conventional techniques in mycology were followed, and the use of specialized literature for its determination.

Results and conclusions: Morphological description of $Q$. mirabilis, photographs of basidioma and microscopic structures of taxonomic importance are presented. In several Mexican states such as Nayarit, it is necessary to increase the explorations for a better knowledge of the country mycobiota.

Keywords: mycobiota, new record, Nayarit

\section{ARTICLE HISTORY}

Received 18 December 2018 / Accepted 20 June 2019

Published on line16 July 2019

\section{INTRODUCCIÓN}

El género Queletia Fr. se creó con Q. mirabilis en 1872 en honor al micólogo Lucien Quélet, indicada como una especie rara (White, 1901). Long y Stouffer (1943) incluyeron Queletia en la subfamilia Tylostomoideae junto con Tulostoma y Schizostoma, por el estípite fibriloso y hueco que sostiene la gleba, y la forma de inserción entre el estípite y la gleba, que difiere entre estos géneros. Zeller (1949) lo incluyó en Tulostomatales junto con Battarrea, Chlamydopus, Phellorinia,

\section{CORRESPONDING AUTOR}

\. Olivia Rodríguez Alcántar, olivia.rodriguez@academicos.udg.mx ORCID: 0000-0002-4525-2952

Schizostoma y Tulostoma. Tradicionalmente, Queletia se clasificó dentro de Sclerodermatales en Tulomastaceae junto con Battarraea, Battarraeoides, Chlamydopus, Phellorinia, Schizostoma y Tulostoma (Moravec, 1958).

Domínguez de Toledo (1993) describió que en Tulostomatales, el estípite puede continuarse con el peridio como en Phellorinia o inserto en una depresión conspicua o inconspicua del saco esporífero llamado acetábulo como en Tulostoma y Queletia, además del margen externo de la depresión como un "collar" en- 
tero a laciniado. La dehiscencia del píleo es el carácter principal disímil entre Queletia, Schizostoma y Tulostoma, siendo irregular, fisura radial esteliforme y a través de un estoma, respectivamente (Long y Stouffer, 1943; Zeller, 1949).

El objetivo del presente estudio es registrar por primera vez el género Queletia para la micobiota mexicana.

\section{MATERIALES Y MÉTODOS}

El material estudiado fue colectado como parte de las exploraciones botánicas realizadas por R. Ramírez-Delgadillo† en Nayarit, en un área con vegetación riparia cercana al arroyo El Reito (Figura 1) y se encuentra depositado en la Colección Micológica del Herbario IBUG. Su análisis micromorfológico se basó en la elaboración de preparaciones de la gleba en hidróxido de potasio al $3 \%$, para la observación de esporas y capilicio bajo un microscopio óptico Carl Zeiss K7. Se midieron en promedio 30 esporas incluida la ornamentación. Las microfotografías se realizaron bajo el programa Axiovision 2.4. La determinación del mate- rial fue con bibliografía especializada (White, 1901; ZeIler, 1949; Domínguez de Toledo, 1993; Pegler et al., 1995). Se siguió la clasificación taxonómica de Kirk et al. (2008) y para la validación, autores y nomenclatura se consultó el Index Fungorum (2018). Se presenta un mapa con la localidad de colecta, la descripción de la especie, fotografías del basidioma, esporas y capilicio.

\section{RESULTADOS}

\section{Descripción de la especie}

Queletia mirabilis Fr., Öfvers. K. Svensk. Vetensk.-Akad. Förhandl. 28: 171 (pl. 4) (1872)

Figura 2

Basidioma epigeo, pileado-estipitado, con píleo globoso a subgloboso de $4.5-5 \mathrm{~cm}$ de diámetro, endoperidio caduco, liso, con dehiscencia apical de forma irregular, de color marrón. Gleba pulverulenta, de color marrón al madurar, sin la presencia de ostiolo. Estípite de 10-15 × 1-2 cm, cilíndrico adelgazado hacia la base, con la superficie fuertemente fibriloso-escamoso, flexuoso, frágil con apariencia de paja cuando

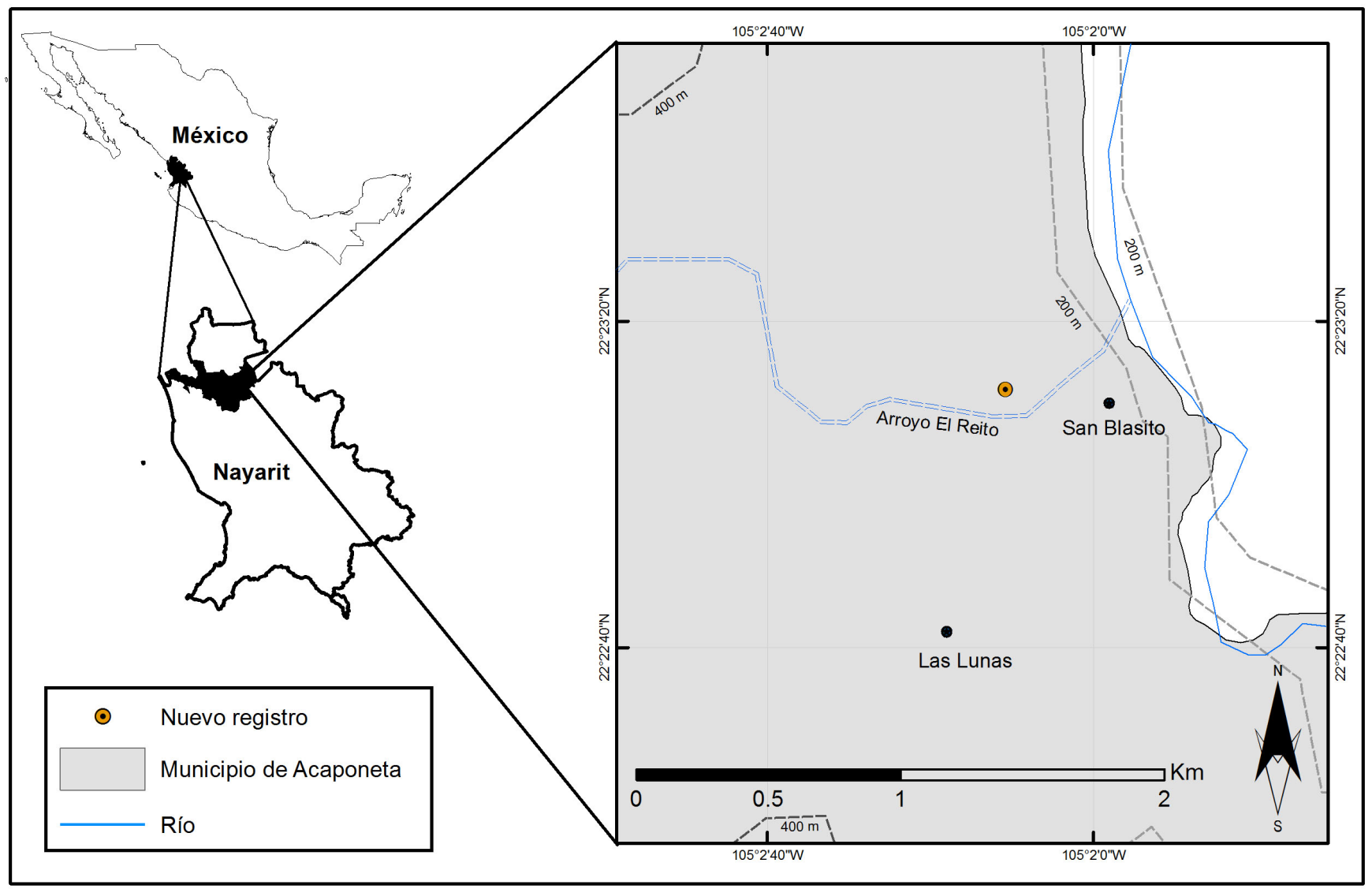

Figura 1. Ubicación geográfica del sitio de colecta. 
seco, de color blanquecino-amarillento con restos de pulverulencia por la presencia de esporas de color marrón, en el ápice sostiene al saco esporífero por medio de un acetábulo, el cual forma un collar con margen laciniado.

Esporas de 7.6-10 × 7.2-9.6 $\mu \mathrm{m}$ ornamentación inclui$\mathrm{da}, \mathrm{Q}=1$ 1-1.09, globosas a subglobosas, equinuladas, con espinas cónicas y apariencia nodulosa por la agrupación de las espinas, de color anaranjado-amarillento. Capilicio de 7.2-13.6 $\mu \mathrm{m}$ de diámetro, abundante, cilíndrico, liso, simple o poco ramificado de forma helicoidal con los extremos obtusos o redondeados, de pared gruesa de $0.8 \mu \mathrm{m}$, de color anaranjado-amarillento, sin septos ni fíbulas.

Material revisado. NAYARIT, Municipio de Acaponeta, arroyo El Reito, al NE de San Blasito, R. Ramírez-Delgadillo s.n., junio 3, 2009 (IBUG).
Hábitat. Gregario, vegetación riparia, bosque tropical caducifolio, altitud 195 m s.n.m.

\section{DISCUSIÓN}

El ejemplar estudiado concuerda con lo descrito por Moreno et al. (2012), en cuanto a forma, color y dehiscencia del basidioma, el tamaño de las esporas y la forma del capilicio, el cual describen de pared gruesa, poco ramificado, cilíndrico de forma helicoidal, sin fíbulas y rara vez septado. Pegler et al. (1995), describen la especie con esporas de 7.5-9.5 $\mu \mathrm{m}$ incluyendo ornamentación, globosas a subglobosas, con verrugas cónicas, de color marrón-amarillento y con capilicio hialino, liso, simple o ramificado, con las puntas obtusas con lumen y septos espaciados. La descripción del capilicio difiere por el color anaranjado-amarillento

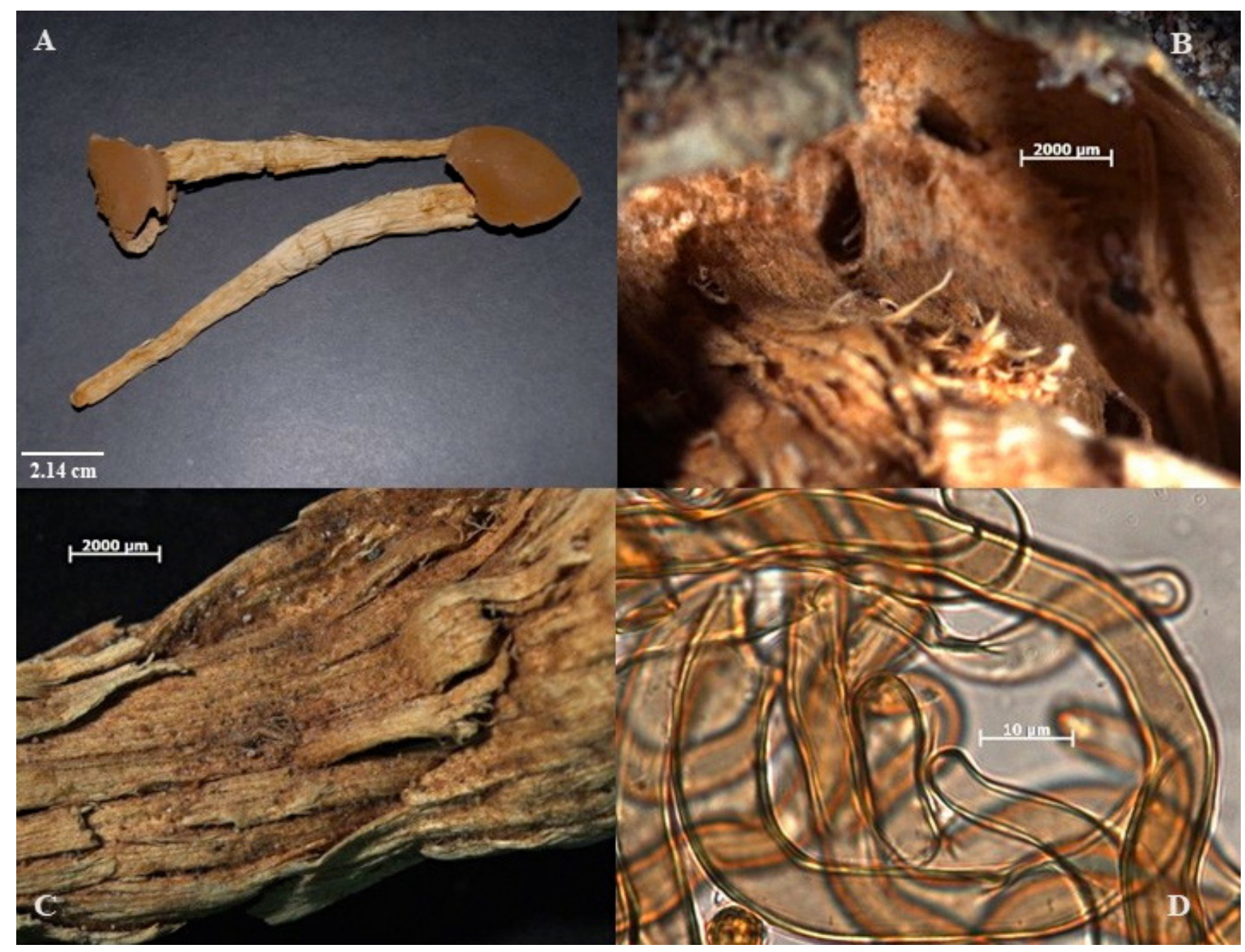

FIGURA 2. Queletia mirabilis, (ejemplar R. Ramírez-Delgadillo s.n., IBUG). A: Basidiomas. B: Acetábulo con margen laciniado. C: Estípite fibriloso-escamoso. D: Capilicio helicoidal con extremos obtusos. 
y sin septos en el material mexicano. Kř́rž y Zíta (2016) describen esporas de (5.7-) 6-8.5 (-9.5) × (5.3-) 5.8-7.5 (-8) $\mu \mathrm{m}$, globosas a subglobosas, ornamentadas por verrugas y espinas, y con capilicio abundante, cilíndrico, poco ramificado de pared gruesa.

La otra especie reconocida es Queletia andina J.E. Wright, caracterizada por su capilicio escaso, poco ramificado y con septos raros o ausentes; esporas lisas de (4.4-) 5.5-6.3 (-7.2) × 3.6-5.2 $\mu \mathrm{m}$, la mayoría elipsoides a globosas, muchas poligonales, de pared gruesa (Wright, 1989). Aunque Queletia mirabilis es una especie poco común, resulta fácil distinguirla por su basidioma estipitado con la superficie fibrilosa-escamosa y el tipo de dehiscencia. Schizostoma es macroscópicamente afín, pero su espora lisa y la dehiscencia radial esteliforme permiten reconocerla fácilmente (Moreno et al., 2012). Registrada para Gran Bretaña (Pegler et al., 1995), España (Moreno et al., 2012), Francia (Azéma, 1990), Italia (Saracini, 2005), República Checa (Kř́íz y Zíta, 2016) y Estados Unidos (Coker y Couch, 1928). Se amplía su distribución en Norteamérica al citarse para México, donde es necesario incrementar las exploraciones para un mejor conocimiento de la micobiota del país. Asimismo, se requieren estudios moleculares de géneros poco estudiados como Queletia, que permitan aclarar su ubicación taxonómica.

\section{AGRADECIMIENTOS}

La primera autora reconoce el apoyo de la Universidad de Guadalajara a través del proyecto P3E 2017-234981 del Cuerpo Académico CA-48, lo cual permitió llevar a cabo este trabajo. Se agradece el apoyo por la elaboración del mapa a Guadalupe Munguía Lino del Laboratorio Nacional de Identificación y Caracterización Vegetal (Laniveg), Unidad Biogeografía. De igual manera las sugerencias y comentarios de los revisores anónimos para mejorar el manuscrito.

\section{LITERATURA CITADA}

Azéma, R.C., 1990. Queletia mirabilis. Bulletin de la Fédération Mycologique Dauphiné-Savoie 119: 20-22.

Coker, W.C., J.N. Couch, 1928. The gasteromycetes of the Eastern United States and Canada. University of North Carolina Press, Chapel Hill.

Domínguez de Toledo, L., 1993. Gasteromycetes (Eumycota) del centro y oeste de la Argentina. I. Análisis crítico de los caracteres taxonómicos, clave de los géneros y orden Podaxales. Darwiniana 32(1-4): 195-235.

Index Fungorum, 2018. Index Fungorum Base de Datos. Disponible en: http://www.indexfungorum.org./Names/Names.asp [consultado 7.X.2018].

Kirk, P.M., P.F. Cannon, D.W. Minter, J.A. Stalpers, 2008. Ainsworth \& Bisby's Dictionary of the fungi. CAB International, Wallingford.

Krŕž, M., V. Zíta, 2016. First records of Gasteromycete Queletia mirabilis in the Czech Republic. Czech Mycology 68(1): 85-95.

Long, W.H., D.J. Stouffer, 1943. Studies in the Gasteromycetes VII: The genus Schizostoma. Mycologia 35(1): 21-32. DOI: $10.2307 / 3754966$

Moravec, Z., 1958. Queletia Fr. -queletovka.- In: Pilât, A. (ed.), Flora CSR [Flora of the Czechoslovak Republic], Ser. B, Fasc. 1, Gasteromycetes, Houby - Brichatky [Gasteromycetes, Gasteroid Fungi]. Nakladatelství CSAV, Praha. Pp. 615-617.

Moreno, G., M.A. Ribes, V. Escobio, A.N. Khalid, 2012. Queletia mirabilis, a rare gasteroide species in Gran Canaria, Spain. Mycotaxon 119: 89-94. DOI: 10.5248/119.89

Pegler, D.N., T. Laessoe, B.M. Spooner, 1995. British puffballs, earthstars and stinkhorns. An account of the British gasteroid fungi. Royal Botanic Gardens, Kew.

Saracini, M., 2005. Gasteromyceti epigei. Fondazione Centro Sttudi Micologici Associazione Micologica Bresadola, Trento.

White, V.S., 1901. The Tylostomaceae of North America. Bulletin Torrey Botanical Club 28(8): 421-444. DOI: 10.2307/2478590

Wright, J.E., 1989. South American Gasteromycetes. III. The genus Queletia Fr. Criptogamic Botany 1: 26-31.

Zeller, S.M., 1949. Keys to the orders, families and genera of the Gasteromycetes. Mycologia 41(1): 36-58. DOI: 10.2307/3755271 